\title{
Primary Metastatic Breast Cancer: The Impact of Locoregional Therapy
}

\author{
Steffi Hartmann Toralf Reimer Bernd Gerber Angrit Stachs
}

Department of Obstetrics and Gynecology, University of Rostock, Germany

Keywords

Breast cancer - Metastatic - Surgery · Radiotherapy · Primary tumor

\section{Summary}

The impact of treatment for the primary tumor on distant metastases and survival in primary metastatic breast cancer patients is controversial. Previous retrospective studies and meta-analyses suggested a survival benefit for the removal of the primary tumor. Early follow-up data from 2 prospectively randomized trials presented at San Antonio Breast Cancer Symposium 2013 could not confirm this. Only a very small subgroup of patients with solitary bone metastases seemed to profit from surgery, while patients with multiple visceral metastases showed a worse prognosis with initial surgery. There are no studies available with the primary aim to investigate the impact of axillary lymph node surgery or locoregional radiotherapy on the survival of stage IV breast cancer patients. Based on current data, locoregional treatment in primary metastatic breast cancer should not be recommended in patients with asymptomatic primary tumor as a matter of routine. More solid conclusion of the impact of primary tumor treatment in stage IV breast cancer patients on their prognosis will be reached with the completion of the ongoing prospectively randomized trials. Until these studies are completed, locoregional therapy, which can provoke additional morbidity in a metastatic setting with limited live expectancy, is exclusively indicated for palliative reasons.

\section{Introduction}

Of all breast cancer patients, $5-10 \%$ present with stage IV disease at the time of primary diagnosis [1]. The 5-year overall survival (OS) in these patients reaches $20 \%$ (historical median survival 16-29 months), and 2-5\% are long-term survivors [2-4]. Treatment goals in stage IV disease are prolongation of survival, control of tumor burden, reduction of cancerrelated symptoms and maintenance of quality of life. The role of the treatment of the primary cancer and its impact on distant metastases and survival is controversial. In other aggressive metastatic cancers like ovarian, renal, colorectal and gastric cancer, an association between decrease in tumor burden and improvement of survival has been shown in the past [5-14]. There are different theories to explain this benefit. It was shown that resection of the primary tumor can remove tumor-induced immunosuppression, stops self-seeding of primary tumor cells to distant sites and may remove a source of potentially chemoresistant cell lines [15-19]. By contrast, animal models demonstrated that removing the primary tumor can stimulate metastatic growth [20-22]. In humans, general anesthesia and surgery decrease immune response [23].

The current National Comprehensive Cancer Network (NCCN) guidelines recommend surgery of the primary tumor after systemic therapy only for patients requiring palliation of symptoms, if complete local clearance of tumor can be obtained and if other sites of disease are not immediately threatening to life [24]. The German Gynecological Oncology Group (Arbeitsgemeinschaft Gynäkologische Onkologie, AGO) guidelines allow surgical resection of the primary tumor and axillary lymph nodes in individual stage IV cases without further characterization of whether this could benefit the patient. The guidelines give no recommendation regarding locoregional radiotherapy (fig. 1) [25]. At present, the dogma for the treatment of metastatic breast cancer is that survival is determined by metastatic disease burden and that local therapy does not affect survival [26]. On the one hand,

\section{KARGER \\ Fax +497614520714 \\ Information@Karger.com}

www.karger.com (c) 2014 S. Karger GmbH, Freiburg

1661-3791/14/0091-0023\$39.50/0

Accessible online at:

www.karger.com/brc 
the morbidity caused by local therapy procedures may add to the burden of suffering in these women. On the other, the indications for local treatment should be reevaluated in light of the advances in systemic therapy with improving survival of stage IV patients $[27,28]$ and very sensitive imaging modalities that help in identifying patients with a very low systemic tumor burden.

This clinical review aims to summarize the current literature dealing with local therapy in stage IV breast cancer patients. On this basis we try to specify the profile of patients that could benefit from locoregional surgery and/or radiotherapy.

\begin{tabular}{|ll|}
\hline Local excision (R0) of primary tumor & $+/-$ \\
Mastectomy & $+/-$ \\
Axillary surgery for cN1 & $+/-$ \\
Sentinel node biopsy in cN0 & - \\
$+/-\quad$ This investigation or therapeutic intervention has not \\
$\quad$ shown benefit for patients and may be performed only \\
$\quad$ in individual cases. According to current knowledge \\
$\quad \begin{array}{l}\text { a general recommendation cannot be given. } \\
\text { This investigation or therapeutic intervention can be of } \\
\text { disadvantage for patients and might not be performed. }\end{array}$ \\
\hline
\end{tabular}

Fig. 1. German Gynecological Oncology Group (Arbeitsgemeinschaft Gynäkologische Onkologie, AGO) guidelines: Breast surgery in primary metastatic disease [25].

\section{Surgical Management of the Breast}

A current meta-analysis investigated whether the excision of the primary tumor had an impact on survival in stage IV breast cancer patients. We identified 10 studies (9 retrospective cohort studies, 1 retrospective case control study) including 28,693 patients comparing the outcome between the groups undergoing surgery versus those with no surgery. The 3 -year survival was significantly increased in those undergoing surgery (40 vs. $22 \%$ ) (table 1) [26]. Comparing the type of surgical procedures, no difference in outcome could be found between breast-conserving therapy and mastectomy [29]. Surgery with negative margins seems to improve survival compared to positive margins or no surgery [26]. Although this meta-analysis reveals a clear advantage for primary tumor surgery, these data need to be discussed very critically. The main problem is the retrospective character of all included studies. A matched-pair analysis discovered that a case selection bias may explain most survival advantage of surgery. In $27 \%$ of the stage IV long-term survivors, chart review showed an incorrect tumor stage classification (stage III instead of IV) [30]. Additionally, surgical patients were found to have a more favorable profile because they tended to have smaller tumors, were younger and had fewer metastases (table 1). No differences between surgery versus non-surgery groups could be found regarding location of metastatic disease, tumor grade and hormone receptor status [26]. Furthermore, there is a lack of information regarding the reasons for surgery. The

Table 1. Results of meta-analysis [26]

\begin{tabular}{|c|c|c|c|c|}
\hline Characteristic & Patients, $\mathrm{n}$ & Surgery, \% & No surgery, \% & $\mathrm{p}$ value \\
\hline 3-year survival & 28,693 & 40 & 22 & $<0.001$ \\
\hline \multicolumn{5}{|l|}{ Tumor size } \\
\hline Total & 24,894 & & & $<0.001$ \\
\hline $\mathrm{T} 0 / \mathrm{Tx} / \mathrm{T} 1 / \mathrm{T} 2$ & 14,093 & 63 & 49 & \\
\hline $\mathrm{T} 3 / \mathrm{T} 4$ & 10,801 & 37 & 51 & \\
\hline Tumor grade & & & & ns \\
\hline Total & 6,030 & & 4.9 & \\
\hline 1 & 282 & 4.4 & & \\
\hline 2 & 2,140 & 35.6 & 35.1 & \\
\hline 3 & 3,608 & 60 & 60 & \\
\hline ER & & & & ns \\
\hline Total & 7,216 & & & \\
\hline Positive & 5,158 & 71 & 73 & \\
\hline Negative & 2,058 & 29 & 27 & \\
\hline PR & & & & $\mathrm{ns}$ \\
\hline Total & 6,557 & & & \\
\hline Positive & 3,762 & 58 & 57 & \\
\hline Negative & 2,795 & 42 & 43 & \\
\hline HER2 & & & & $<0.05$ \\
\hline Total & 402 & & & \\
\hline Amplified & 132 & 44 & 26 & \\
\hline Not amplified & 270 & 56 & 74 & \\
\hline Metastasis at: & & & & $<0.001$ \\
\hline Total & 1,888 & & & \\
\hline 1 site & 995 & 63 & 44 & \\
\hline$>1$ site & 839 & 37 & 56 & \\
\hline Metastatic site & & & & ns \\
\hline Total & 2,395 & & & \\
\hline Nonvisceral & 962 & 42 & 39 & \\
\hline Visceral & 1,433 & 58 & 61 & \\
\hline
\end{tabular}


Table 2. Prospective randomized trials investigating the impact of locoregional therapy in stage IV breast cancer patients (modified from [35])

\begin{tabular}{llll}
\hline Trial number/ country & Accrual period & Patients, $\mathrm{n}$ & Primary endpoint \\
\hline NCT00193778/ India & $2005-2012$ & 350 & Final data collection \\
NCT00557986/ Turkey & $2008-2012$ & 278 & June 2016 survival \\
NCT01242800/ USA and Canada & $2011-2016$ & 368 & Noverall survival \\
NCT01392586/ Netherlands & $2011-2016$ & 516 & survival \\
NCT01015625/ Austria & $2010-2019$ & 2012 & 2 -year survival \\
JCOG1017/ Japan & $2011-2016$ & 410 & survival \\
survival & February 2018 \\
\hline
\end{tabular}

$\mathrm{n} / \mathrm{a}=$ not available.

traditional indication for local surgery in stage IV breast cancer patients is palliation (bleeding, ulceration, fungating). Only 3 studies documented the indication for surgery [31-33]. Bafford et al. [34] found a survival benefit only for patients who were operated before the diagnosis of metastatic disease. This could be another source for a selection bias, because these patients with intended curative surgery are suspected to have a lower metastatic tumor burden and, therefore, a better prognosis. Human epidermal growth factor receptor 2 (HER2)status data were limited in most studies. A recent retrospective study investigated the survival of stage IV breast cancer patients with local surgery, and revealed a statistically significantly improved survival only for patients with estrogen receptor/progesterone receptor (ER/PR)-positive or HER2positive disease. No survival benefit was observed in patients with triple-negative (TNBC) disease. They conclude that the impact of local control is greatest in the presence of effective targeted therapy [33].

The pivotal question, which selected patients would benefit from breast surgery concerning survival, cannot be answered by the existing retrospective data nor by the current metaanalysis. The optimal point of time and the decision for or against surgery depending on the response to systemic therapy remain unclear.

Because of the retrospective design of all studies dealing with this topic and the potential biases described above, prospective randomized trials are needed to define the impact of local treatment on survival and to define subsets of patients who would benefit most from surgery. Therefore, 6 prospective randomized studies were initiated worldwide to assess a potential benefit of local therapy in primary metastatic breast cancer (table 2) [35]. 2 studies have now been completed, and their first results were presented at the San Antonio Breast Cancer Symposium (SABCS) 2013. The Turkey MF07-01 trial is a phase III randomized controlled trial comparing patients who received locoregional treatment (mastectomy or breast-conserving therapy + whole breast irradiation, axillary lymph node dissection level I-II when clinically positive or positive sentinel lymph node were present) with those who did not. 278 evaluable patients were recruited (140 women in the surgery group, 138 women in the no-surgery group). After randomization into groups with/without surgery, standard systemic therapy started immediately. According to the study protocoll all patients received chemotherapy, endocrine therapy if hormone receptor-positive, and trastuzumab for
HER2-positive disease. Only bisphosphonate was given at the discretion of the treating physician. After a median follow-up of 18 months, no statistically significant difference for survival rate could be shown between the surgery and no-surgery groups ( 35 vs. $31 \%, p=0.24$ ). The distribution of biological subtypes were as follows: ER/PR positive $86 \%$ (initial surgery) vs. $72.3 \%$ (systemic treatment), HER2postive $30.7 \%$ vs. $30.4 \%$ and TNBC $7.1 \%$ vs. $17.4 \%$. According to these data, there was a trend for more endocrineresponsive carcinomas and fewer TNBC in the surgery group, but differences were not statistically significant. Surgery in the subgroup of patients with solitary bone-only metastases had statistically significant survival benefit compared with no surgery, while patients with multiple liver and/or lung metastases had a significant worse prognosis with initial surgery [36]. The second completed prospective randomized trial from Indian Tata Memorial Centre randomized 350 patients after an initial 6 cycles of anthracycline-based chemotherapy into 2 groups (mastectomy or breast-conserving therapy +/irradiation ( $\mathrm{n}=173$ ) vs. no locoregional treatment $(\mathrm{n}=177)$ ). No HER2-positive patient received HER2-targeted therapy. After a median follow-up of 17 months there was no statistical difference for OS between the locoregional treatment group and the group without locoregional treatment (18.8 vs. 20.5 months, $\mathrm{p}=0.60)$. Although the local progression-free survival was significantly higher for the locoregional treatment group, the distant progression-free survival was significantly better for the group with no locoregional treatment. The impressive death rate $(n=268,77 \%)$ indicates recruitment of high-risk carcinomas or highly advanced tumors. It is unclear whether asymptomatic, hormone receptor (HR)-positive patients with indication for endocrine therapy alone were included in the study. Interestingly, subgroup analysis for event rates with respect to histological subtypes revealed identical odds ratios for HR-negative and HR-positive disease (1.10 vs. 1.10) [37].

Neither prospective studies showed a survival benefit for removing the primary tumor in stage IV breast cancer patients. Therefore, at the moment, breast surgery for these patients should not be offered as a routine practice. Removing the primary tumor could confer a growth advantage on distant metastases, especially in women with multiple distant metastases $[36,37]$. These early results confirm the hypothesis of a selection bias in the previous retrospective investigations, which postulated a survival benefit due to surgery. Therefore, 
Table 3. Studies investigating axillary lymph node surgery in stage IV breast cancer patients

\begin{tabular}{lccl}
\hline Author & Patients with/without surgery, & $\begin{array}{l}\text { Patients with axillary lymph node } \\
\text { procedure (\% of surgery patients) }\end{array}$ & $\begin{array}{l}\text { Effect of axillary surgery } \\
\text { on survival }\end{array}$ \\
\hline Babiera et al. 2006 [31] & $49 / 33$ & $49(100)$ & $\mathrm{n} / \mathrm{a}$ \\
Rapiti et al. 2006 [39] & $127 / 173$ & $73(57)$ & $\mathrm{ns}$ \\
Ruiterkamp et al. 2009 [29] & $288 / 440$ & $190(66)$ & $\mathrm{ns}$ \\
Fields et al. 2007 [32] & $187 / 222$ & $151(81)$ & $\mathrm{n} / \mathrm{a}$ \\
Hazard et al. 2008 [38] & $47 / 64$ & $31(66)$ & $\mathrm{n} / \mathrm{a}$ \\
Neuman et al. 2010 [33] & $69 / 117$ & $33(48)$ & $\mathrm{n} / \mathrm{a}$ \\
\hline
\end{tabular}

$\mathrm{n} / \mathrm{a}=$ not available, $\mathrm{ns}=$ not significant.

although local therapy at the primary site may provide a local control advantage, a possible survival advantage currently remains unproven, and surgery of the primary asymptomatic tumor should not be offered to patients outside the setting of a clinical trial. When all 6 prospectively randomized trials are completed, we will be able to reach a more solid conclusion of the impact of primary tumor surgery on prognosis in primary metastatic breast cancer patients. The very small subgroup of patients with solitary bone-only metastases seems to be only one that could benefit from breast surgery at the moment [36].

\section{Axillary Lymph Node Dissection}

No studies with the primary aim of comparing survival data due to axillary lymph node management in stage IV breast cancer patients were found. 6 retrospective studies gave information on whether an axillary surgical procedure was performed in case of surgery (table 3). Altogether, 1,816 patients were reviewed, of whom 767 (42\%) underwent surgery. In the surgery group, 527 patients $(69 \%)$ had axillary procedures (axillary lymph node dissection in 493 patients (93\%), sentinel lymph node biopsy in 34 patients (7\% )) [29, 31-33, 38, 39]. Only 3 studies investigated the impact of axillary surgery on survival, and did not find a benefit [29, 35]. The purpose for conducting axillary dissection was evaluated by only 1 study; $84 \%$ of the patients underwent axillary lymph node dissection before the diagnosis of metastases. They underwent metastatic surveys after surgery because of 4 or more pathologically positive lymph nodes [38]. The rate of axillary lymph node recurrence after axillary lymph node clearance was found to be $9 \%$, and is somewhat higher than that reported in the non-metastatic setting [33]. None of the completed or ongoing prospective trials dealing with local therapy in women with metastatic breast cancer aimed to evaluate the effect of axillary lymph node dissection on their prognosis.

Currently, we have only very few retrospective data that suggest that axillary lymph node dissection is not beneficial. Because of possible side effects such as edema, pain and limitation of arm mobility, it should not be a routine procedure in metastatic breast cancer patients with limited life expectancy to avoid additional suffering. The value of axillary surgery with respect to survival and quality of life in stage IV breast cancer patients should be investigated in future studies.

\section{Locoregional Radiotherapy}

The role of local radiotherapy in patients with metastatic breast cancer is largely unexplored. There have been no prospective randomized trials dealing with this topic. In retrospective studies and large databases such as SEER (Surveillance, Epidemiology, and End Results), it is often not possible to differentiate between radiation of the primary site from that of the metastatic lesion [40]. In stage IV disease, radiotherapy of the primary is frequently combined with surgery, making it difficult to discriminate between effects of surgery or radiotherapy alone. In terms of locoregional control, primary radiotherapy seems to be as effective as surgery. In a retrospective study by Bourgier at al. [41], radiation alone was compared with surgery of the primary plus radiotherapy. The 3 -year metastasis progression-free survival were $20 \%$ and $39 \%$, 3-year OS rates were $39 \%$ and $57 \%$. No significant differences were found when adjusted to prognostic factors. Le Scodan et al. [42] presented a retrospective review of 581 patients with primary metastatic breast cancer at the Renee Cancer Center in France. Locoregional therapy (LRT) was applied to 320 patients: 249 (78\%) were treated with radiotherapy alone, $41(13 \%)$ underwent surgery plus adjuvant radiation, and $30(9 \%)$ were treated only with surgery. The 3 -year OS rates were $43.2 \%$ in the group with LRT and $26.7 \%$ in the group without LRT $(\mathrm{p}=0.0002)$. However, patients in the group with LRT had a lower T stage, were more likely to have non-visceral metastases, and had fewer metastatic sites compared with the group without LRT. Nevertheless, the survival benefit of LRT was confirmed by multivariate analysis with a risk reduction of death of $30 \%$ (multi-adjusted hazard ratio $=0.70 ; 95 \%$ CI $0.58-0.85)$. In subgroup analysis, patients with only bone metastases did not benefit from additional LRT. This may be due to the fact that patients with bone-only metastases frequently have receptor-positive disease, which tends to have a more indolent course. Surprisingly, in the article by Le Scodan et al., the survival benefit for patients with multiple visceral metastases was similar to that of the entire study population with a median survival time of 25 months and 3-year OS of $34.2 \%$ for patients with LRT in comparison to 13 months and $17.8 \%$ for patients without LRT. This is contrary to retrospective studies dealing with surgery of the primary in stage IV breast cancer, which revealed disadvantage in terms of survival for patients with high visceral tumor 
burden [36]. The presented retrospective data may challenge the dogma of reserving local radiation therapy only for palliative purposes. However, interpretation of such retrospective non-randomized trials has to be treated with caution, since selection bias has to be taken into account. Therefore, the role of radiation therapy of the breast and lymph nodes in case of synchronous metastases remains quite indefinite.

\section{Conclusions}

Traditionally, in stage IV breast cancer patients LRT has been preserved for palliation and symptom control. Retrospective observational studies revealed that about $60 \%$ of patients with primary metastatic disease are treated locally, leading to a survival benefit in most studies. Taking into account that stage IV breast cancer patients are a heterogeneous population with regard to molecular subtype, age, overall tumor burden, visceral involvement and systemic treatment response, the limitations of data generated from retrospective studies are obvious. First data from 2 prospective randomized trials presented at SABCS 2013 demonstrate no survival benefit for patients treated with LRT compared to the group without LRT. Patients with only bone metastases could be a subgroup qualifying for LRT since retrospective as well as prospective data reveal a survival benefit in this constellation.

With the introduction of new systemic treatment agents breast cancer outcomes are improving steadily. Therefore, we have to consider stage IV breast cancer more as a chronic disease than a terminal event. For that reason, the first step to treat metastatic breast cancer should be systemic therapy. If a response is seen at all sites, continuation of the effective systemic therapy is reasonable. In patients whose tumor is well controlled at distant sites but progressing locally, local surgery and/or radiotherapy should be discussed. There are still open questions with regard to axillary treatment and indication for radiotherapy. The ongoing prospective randomized trials dealing with LRT in stage IV breast cancer need to be completed. Essentially, LRT should not be offered to patients with asymptomatic tumors outside of clinical trials.

\section{Disclosure Statement}

The authors declare that there are no conflicts of interest related to this study.

\section{References}

1 Howlader N, Noone AM, Krapcho M, et al. (eds): SEER cancer statistics review. Bethesda: National Cancer Institute, 1975-2008. http://seer.cancer.gov/ csr/1975_2008/.

2 Carmichael AE, Anderson ED, Chetty U, Dixon JM: Does local surgery have a role in the management of stage IV breast cancer? Eur J Surg Oncol 2003;29:2011-2019.

3 Cardoso F, Castiglione M, ESMO Guidelines Working Group: Locally recurrent or metastatic breast cancer: ESMO clinical recommendations for diagnosis, treatment and follow-up. Ann Oncol 2009;20 Suppl 4:15-18.

4 Surveillance, Epidemiology, and End Results (SEER) Program (www.seer.cancer.gov). SEER*Sta Database: Mortality - all COD, Aggregated with State, Total U.S. (1969-2010) <Katrina/Rita population adjustment $>$, National Cancer Institute, DCCPS, Surveillance Research Program, Surveillance System Branch, released April 2013. underlying mortality data provided by NCHS (www. cdc.gov/nchs).

$\checkmark 5$ Griffith CT, Fuller AR: Intensive surgical and chemotherapeutic management of advanced ovarian cancer. Symposium on gynecologic cancer. Surg Clin North Am 1978;58:131-142.

6 Dauplat J, Le Bouedec G, Pomel C, Scherer C: Cytoreductive surgery for advanced stages of ovarian cancer. Semin Surg Oncol 2000;19:42-48.

7 Chi, DS, Eisenhauer EL, Lang J, et al.: What is the optimal goal of primary cytoreductive surgery for bulky stage IIIC epithelial ovarian carcinoma (EOS)? Gynecol Oncol 2006;103:559-564.

8 Griffith CT, Parker LM, Lee, S, Finkler NJ: The effect of residual mass size on response to chemotherapy after surgical cytoreduction for advanced ovarian cancer: long-term results. Int J Gynecol Cancer 2002;12:323-331.
9 Flanigan RC, Salmon SE, Blumenstein BA, et al.: Nephrectomy followed by interferon alfa- $2 \mathrm{~b}$ compared with interferon alfa- $2 b$ alone for metastatic renal cell cancer. N Engl J Med 2001;345:1655-1659.

10 Rosen SA, Buell JF, Yoshida A, et al.: Initial presentation with stage IV colorectal cancer: How aggressive should we be? Arch Surg 2000;135:530534

11 Martin R, Paty P, Fong Y, et al.: Simultaneous liver and colorectal resections are safe for synchronous colorectal liver metastasis. J Am Coll Surg 2003; 197:233-241.

12 Abdalla EK, Vauthey JN, Ellis LM, et al.: Recurrence and outcomes following hepatic resection, radiofrequency ablation, and combined resection/ ablation for colorectal liver metastases. Ann Surg 2004:239:818-825.

13 Tanaka K, Shimada H, Matsuo K, et al.: Outcome after simultaneous colorectal and hepatic resection for colorectal cancer with synchronous metastases. Surgery 2004;136:650-659.

14 Hallissey MT, Allum WH, Roginski C, Fielding JWL: Palliative surgery for gastric cancer. Cancer 1988;62:440-444.

15 Danna EA, Sinha P, Gilbert M, et al.: Surgical removal of the primary tumor reverses tumorinduced immunosuppression despite the presence of metastatic disease. Cancer Res 2004;64:2205-2211.

16 Norton L, Massague J: Is cancer a disease of selfseeding? Nat Med 2006;12:875-878.

17 Cristofanilli M, Budd GT, Ellis MJ, et al.: Circulating tumor cells, disease progression, and survival in metastatic breast cancer. N Engl J Med 2004;351: 781-791.

18 Arriagada R, Rutquist LE, Mattsson A, et al.: Adequate locoregional treatment for early breast cancer may prevent secondary dissemination. J Clin Oncol 1995;13:2869-2878.
19 Goldie JH, Coldman AJ: A mathematic model for relating the drug sensitivity of tumors to their spontaneous mutation rate. Cancer Treat Rep 1979;63:1727-1733.

20 Fisher B, Gunduz N, Coyle J, et al.: Presence of a growth-stimulating factor in serum following primary tumor removal in mice. Cancer Res 1989; 49:1996-2001.

21 O'Reilly MS, Holmgren L, Shing Y, et al.: Angiostatin: A novel angiogenesis inhibitor that mediates the suppression of metastases by a Lewis lung carcinoma. Cell 1994;79:315-328.

22 Gunduz N, Fisher B, Saffer EA: Effect of surgical removal on the growth and kinetics of residual tumor. Cancer Res 1979;39:3861-3865.

23 Salo, M: Effects of anaesthesia and surgery on the immune response. Acta Anaesthesiol Scand 1992; 36:201-220.

24 Theriault RL, Carlson RW, Allred C, et al.: Breast cancer, version 3, 2013: Featured updates to the NCCN guidelines. J Natl Compr Canc Netw 2013; 11:753-760.

25 Members of the AGO Breast Committee: Diagnosis and treatment of patients with primary and metastatic breast cancer. Guidelines of the AGO Breast Committee 2013.

26 Harris E, Barry M, Malcolm RK: Meta-analysis to determine if surgical resection of the primary tumour in the setting of stage IV breast cancer impacts on survival. Ann Surg Oncol 2013;20:28282834.

27 Giordano SH, Buzdar AU, Smith TL, et al.: Is breast cancer survival improving? Cancer 2004;100: 44-52.

28 Andre F, Slimane K, Bachelot T, et al.: Breast cancer with synchronous metastases: Trends in survival during a 14-year period. J Clin Oncol 2004; 22:3302-3308. 
29 Ruiterkamp J, Ernst MF, van de Poll-Franse LV, et al.: Surgical resection of the primary tumour is associated with improved survival in patients with distant metastatic breast cancer at diagnosis. Eur J Clin Oncol 2009;35:1146-1151.

-30 Cady B, Nathan NR, Michaelson JS, et al. Matched pair analyses of stage IV breast cancer with or without resection of primary breast site. Ann Surg Oncol 2008;15:3384-3395.

-31 Babiera GV, Rao R, Feng L, et al.: Effect of primary tumor exstirpation in breast cancer patients who present with stage IV disease and an intact primary tumor. Ann Surg Oncol 2006; 13:776-782.

32 Fields RC, Jeffe DB, Trinkaus K, et al.: Surgical resection of the primary tumor is associated with increased long-term survival in patients with stage IV breast cancer after controlling for site of metastasis. Ann Surg Oncol 2007:14:3345-3351.

33 Neuman HB, Morrogh M, Gonen M, et al.: Stage IV breast cancer in the era of targeted therapy. Does surgery of the primary tumor matter? Cancer 2010;116:1226-1233
34 Bafford AC, Burstein HJ, Barkley CR, et al.: Breast surgery in stage IV breast cancer: Impact of staging and patient selection on overall survival. Breast Cancer Res Treat 2009;115:7-12.

35 Khan SA, Steward AK, Morrow M: Does aggressive local therapy improve survival in metastatic breast cancer? Surgery 2002;132:620-627.

36 Soran A, Ozmen V, Ozbas S, et al.: Early follow up of a randomized trial evaluating resection of the primary breast tumor in women presenting with de novo stage IV breast cancer; Turkish study (protocol MF07-01). SABCS 2013, General Session 2, Dec 11, S2-03.

37 Badwe R, Parmar V, Hawaldar R, et al.: Surgical removal of primary tumor and axillary lymph nodes in women with metastatic breast cancer at first presentation: A randomized controlled trial. SABCS 2013, General Session 2, Dec 11, S2-02.

38 Hazard HW, Gorla SR, Scholtens D, et al.: Surgical resection of the primary tumor and chest wall control in women with metastatic breast cancer. Cancer 2008;113:2011-2019.
39 Rapiti E, Verkooijen HM, Vlastos G, et al.: Complete excision of primary breast tumor improves survival of patients with metastatic breast cancer at diagnosis. J Clin Oncol 2006;24:2743-2749.

40 Mayadev J, Chen A, Bold R, et al.: The potential role of radiation therapy to the primary site of disease in stage IV breast cancer presenting with synchronous metastasis. Clin Breast Cancer 2014; 14:10-12.

41 Bourgier C, Khodari W, Vataire A, et al.: Breast radiotherapy as part of loco-regional treatments in stage IV breast cancer patients with oligometastatic disease. Radiother Oncol 2010;96:199-203.

42 Le Scodan R, Stevens D, Brain E, et al.: Breast cancer with synchronous metastases: survival impact of exclusive locoregional radiotherapy. J Clin Oncol 2009;27:1375-1381. 